\title{
In Vitro Characterization of the Cytochrome P450 Isoforms Involved in the Metabolism of 6-Methoxy-2-napthylacetic Acid, an Active Metabolite of the Prodrug Nabumetone
}

\author{
Kaori Matsumoto, ${ }^{a}$ Eiichi Nemoto, ${ }^{a, b}$ Tetsuya Hasegawa, ${ }^{a}$ Masayuki Aкimoto, ${ }^{*, a}$ and \\ $\mathrm{Kenji} \mathrm{SugIBAYASHI}^{b}$ \\ ${ }^{a}$ Faculty of Pharmaceutical Sciences, Josai International University; 1 Gumyo, Togane, Chiba 283-8555, Japan: and \\ ${ }^{b}$ Faculty of Pharmaceutical Sciences, Josai University; 1-1 Keyakidai, Sakado, Saitama 350-0295, Japan. \\ Received October 14, 2010; accepted February 15, 2011; published online February 18, 2011
}

The cytochrome P450 (CYP) isoforms that catalyze the oxidation metabolism of 6-methoxy-2-napthylacetic acid (6-MNA), an active metabolite of nabumetone, were studied in rats and humans. Using an extractive reversed-phase HPLC assay with fluorescence detection, monophasic Michaelis-Menten kinetics was obtained for the formation of 6-hydroxy-2-naphthylacetic acid (6-HNA) in liver microsomes of rats and humans, and kinetic analysis showed that the $K_{\mathrm{m}}$ and $V_{\max }$ values for the formation of 6-HNA in humans and rats were $640.0 \pm 30.9$ and $722.9 \pm 111.7 \mu \mathrm{M}$, and $1167.5 \pm 33.0$ and $1312.7 \pm 73.8 \mathrm{pmol} \mathrm{min}^{-1} \mathrm{mg} \mathrm{protein}^{-1}$, respectively. The CYPs responsible for metabolism of 6-MNA in liver microsomes of rats and humans were identified using correlation study, recombinant CYP supersomes, and specific CYP inhibitors and antibodies. Recombinant human CYP2C9 exhibited appreciable catalytic activity with respect to 6-HNA formation from 6-MNA. Among 14 recombinant rat CYPs examined, CYP2C6, CYP2C11 and CYP1A2 were involved in the metabolism of 6-MNA. Sulfaphenazole (a selective inhibitor of CYP2C9) inhibited the formation of 6-HNA in pooled human microsomes by $\mathbf{8 9} \%$, but failed to inhibit this reaction in rat liver microsomes. The treatment of pooled human liver microsomes with an antibody against CYP2C9 inhibited the formation of 6-HNA by about $80 \%$. The antibody against CYP2C11 suppressed the activity by 20 to $30 \%$ in rat microsomes, whereas that of CYP1A2 microsomes did not show drastic inhibition. These findings suggest that CYP2C9 has the highest catalytic activity of 6-MNA metabolism in humans. In contrast, metabolism of 6-MNA is suggested to be mediated mainly by CYP2C6 and CYP2C11 in rats.

Key words nabumetone; metabolite; liver microsome; human; rat; cytochrome P450

Nabumetone, 4-(6-methoxy-2-naphthyl)-butan-2-one, is a nonsteroidal anti-inflammatory drug (NSAID) that has proved effective in the treatment of rheumatoid and osteoarthritis. ${ }^{1)}$ Nabumetone is a prodrug that undergoes extensive first-pass metabolism to 6-methoxy-2-naphthylacetic acid (6-MNA), the major circulating metabolite. 6-MNA is largely responsible for the therapeutic efficacy of nabumetone. It decreases prostaglandin synthesis via inhibition of cyclooxygenase, an enzyme involved in the arachidonic acid conversion pathway. ${ }^{2)}$

The metabolism of 6-MNA has been studied in rats, the major experimental animal, and humans. The primary metabolite of 6-MNA, namely, 6-hydroxy-2-naphthylacetic acid (6-HNA), was detected in urine. About $70 \%$ of a radiolabeled dose of nabumetone was recovered within $48 \mathrm{~h}$ in urine, mainly as 6-MNA, 6-HNA and their conjugates. ${ }^{3)}$ The 6-O-demethylation of 6-MNA to 6-HNA is known to be a major metabolic pathway (Fig. 1). Although it may be obvious that the cytochrome P450 (CYP) system is involved in this pathway, actual CYP isoforms that are involved have not been identified. Their identification is of considerable clinical importance with regard to potential drug interactions and ge-

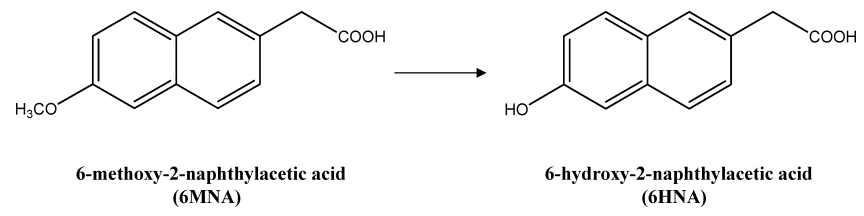

Fig. 1. Chemical Structures of 6-MNA and 6-HNA netically based individual variations of drug metabolism. To our knowledge, no in vitro study has been conducted with respect to the metabolism of 6-MNA or to obtain specific data on the enzyme(s) involved in its metabolic pathway in vitro using human and rat liver microsomes.

The purpose of this study was to elucidate CYP isoforms with regard to the 6-O-demethylase activity, a major metabolic pathway of 6-MNA, in human/rat microsomes and recombinant human CYP enzymes expressed in insect cells in which both human/rat CYP and reduced nicotinamide adenine dinucleotide phosphate (NADPH)-P450 reductase cDNAs had been incorporated. We also examined the inhibition or activation of 6-MNA metabolism by chemical substrates and antibodies.

\section{MATERIALS AND METHODS}

Chemicals and Reagents 6-MNA and 6-HNA were supplied by Sanwa Kagaku Kenkyusho (SKK) Co., Ltd. (Nagoya, Japan). Glucose-6-phosphaste and glucose-6-dehydrogenase were obtained from Oriental Yeast (Tokyo, Japan). NADPH and nicotinamide adenine dinucleotide phosphate (NADP) were purchased from Alexis (San Diego, CA, U.S.A.). Mefenamic acid and naproxen were purchased from Tokyo Kasei Kogyo Co. (Tokyo, Japan). Acetonitrile, methanol and cimetidine were purchased from Wako Pure Chemical Industries Ltd. (Tokyo, Japan). Furafylline, troleandomycin, ketoconazole, sulfaphenazole and $S$-mephenytoin were purchased from Daiichi Pure Chemicals (Tokyo, Japan). Diethyldithiocarbamate and quinidine were pur- 
chased from Sigma Chemical Co. (St Louis, MO, U.S.A.). Diclofenac and 4'-hydoroxydiclofenac were purchased from LKT Laboratories, Inc. (St. Paul, MN, U.S.A.) and Calbiochem (Darmstadt, Germany), respectively. Polyclonal antibodies against human $\mathrm{CYP} 2 \mathrm{C} 9$ and against rat CYP1A2, CYP2C11 and NADPH-P450 reductase were purchased from Nippon Nosan Kogyo Co., Ltd. (Yokohama, Japan). Other chemicals and reagents used in this study were obtained from sources described previously or were of the highest qualities commercially available.

Enzyme Preparations Microsomes from baculovirusinfected insect cells expressing CYP1A1, CYP1A2, CYP2A1, CYP2A2, CYP2A6, CYP2B1, CYP2B6, CYP2C6, CYP2C8, CYP2C9, CYP2C11, CYP2C12, CYP2C13, CYP2C19, CYP2D1, CYP2D2, CYP2D6, CYP2E1, CYP3A1, CYP3A2, CYP3A4 and CYP4A11 were obtained from BD Gentest Co. (Woburn, MA, U.S.A.). These were coexpressed with NADPH-CYP oxidoreductase. Individual human liver microsomes (HG3, HG74, HG95, HH13, HH47 and HK37) were also purchased from BD Gentest Co. (Woburn, MA, U.S.A.). Pooled human liver microsomes (HMMC-PL020) were purchased from Cosmo Bio Co., Ltd. (Tokyo, Japan). The pooled human liver microsomes were obtained from 15 donors. The immunochemically determined CYP contents and specific catalytic activities of each CYP isoform in these microsomes were provided in the data sheets of the manufacturer. Pooled liver microsomes from male and female Sprague-Dawley rats were also purchased from BD Gentest Co. (Woburn, MA, U.S.A.).

6-MNA-O-Demethylase Activity The 6-MNA-O-demethylase activities in liver microsomes or microsomes from the expression system were determined by HPLC with fluorescence detection as described by Kobylinska et al. ${ }^{4)}$ with slight modification. The standard incubation conditions were chosen on the basis of the results of preliminary experiments in which concentrations of both substrate and microsomal proteins were varied. A typical incubation mixture $(0.2 \mathrm{ml}$ of total volume) contained $50 \mathrm{~mm}$ phosphate buffer $(\mathrm{pH} 7.4)$, an NADPH-generating system $\left(0.5 \mathrm{~mm} \mathrm{NADP}^{+}, 0.5 \mathrm{~mm} \mathrm{NADPH}\right.$, $5 \mathrm{~mm}$ glucose-6-phosphate, $5 \mathrm{~mm} \mathrm{MgCl}_{2}, 1 \mathrm{IU} / \mathrm{ml}$ glucose-6phosphate dehydrogenase), 6-MNA and $0.5 \mathrm{mg} / \mathrm{ml}$ microsomal protein of liver. The reaction was initiated by adding liver microsomes after $3 \mathrm{~min}$ preincubation of the NADPHgenerating system at $37^{\circ} \mathrm{C}$. The reaction mixtures were incubated for $40 \mathrm{~min}$ and reactions were terminated by the addition of $280 \mu \mathrm{l}$ of $10 \% \mathrm{HClO}_{4}$ including $100 \mu \mathrm{M}$ naproxen as an internal standard. After removal of the protein by centrifugation at $10000 \mathrm{rpm}$ for $5 \mathrm{~min}$, a $200 \mu \mathrm{l}$ portion of the supernatant was applied to $\mathrm{C} 8+$ strong anion exchange solid-phase extraction columns, which had first been conditioned with $5 \%$ methanol in water. After adsorption of the samples, cartridges were washed with $4 \mathrm{ml}$ of water. Analytes were then eluted with $6 \mathrm{ml}$ of hexane-ethyl acetate $(1: 1)$, evaporated to dryness and reconstituted in $200 \mu \mathrm{l}$ of mobile phase. ${ }^{5)}$ The eluate was injected into the HPLC system for quantification.

For recombinant CYPs, the incubation mixture was of the same composition as mentioned above, except for 200 $\mathrm{pmol} / \mathrm{ml} \mathrm{CYP}$ for microsomes from baculovirus-infected insect cells expressing CYPs and $100 \mu \mathrm{M}$ 6-MNA.

The HPLC system consisted of a Shimadzu LC-10 system equipped with a SPD10-AV fluorescence detector (Shi- madzu, Kyoto, Japan). Chromatography was conducted on a YMC-Pack ODS-A $(150 \times 4.6 \mathrm{~mm}$ i.d.; particle size $=5 \mu \mathrm{m}$; YMC, Kyoto, Japan) eluted at $1.0 \mathrm{ml} / \mathrm{min}$ with the following mobile phase: $\mathrm{CH}_{3} \mathrm{CN} / 20 \mathrm{~mm} \mathrm{~K}_{2} \mathrm{HPO}_{4}(\mathrm{pH} 3.0)=1: 1$. The eluent was monitored at excitation and emission wavelengths of 280 and $350 \mathrm{~nm}$, respectively, and the column temperature was $30^{\circ} \mathrm{C}$.

Correlation Studies CYP2C9 activity was determined with $50 \mu \mathrm{M}$ diclofenac (dissolved in $50 \mathrm{~mm}$ phosphate buffer). The formation of 6-HNA was correlated with diclofenac 4 '-hydroxylation activity in microsomes from 6 different human livers using $200 \mu \mathrm{M}$ 6-MNA.

Chemical Inhibition and Activation Studies The following chemical inhibitors were used: both thiourea and methimazole for flavin-containing monooxigenases (FMOs), both $n$-benzylimidazole (NBI) and SKF525A for CYPs, furafylline for CYP1A2, sulfaphenazole for CYP2C9, $S$ mephenytoin for CYP2C19, quinidine for CYP2D6, diethyldithiocarbamate for CYP2E1, troleandomycin and ketoconazole for CYP3A4, cimetidine for CYP2C11/2C6. ${ }^{6-12)}$ There are reports that thiourea and NBI were highly selective for FMOs and CYPs as compared to methimazole, and SKF525A, respectively. ${ }^{13,14)}$ 6-MNA $(200 \mu \mathrm{M})$ was incubated with and without one or more than one of the inhibitors at concentrations that produced inhibitory effects in excess of $90 \%$ under the incubation conditions described above. 6MNA was also incubated with four concentrations of dapsone, $0-100 \mu \mathrm{M}$, in order to assess degree of activation. ${ }^{15)}$ The effects of each compound on the formation of 6-HNA from 6-MNA at the respective inhibitor concentrations were compared with that with the incubation of 6-MNA alone and expressed as a percentage of the respective control value.

Immunoinhibition Studies The immunoinhibition of 6HNA formation from 6-MNA was examined by preincubating human or rat liver microsomal samples $(200 \mu \mathrm{g})$ with various amounts ( 0 to $50 \mu \mathrm{l})$ of preimmune immunoglobulin $\mathrm{G}$, anti-rat NADPH P450 reductase antibody, anti-CYP2C9 antibody, anti-CYP2C11 antibody and anti-CYP1A2 antibody in $100 \mathrm{~mm}$ Tris- $\mathrm{HCl}$ buffer $(\mathrm{pH} \mathrm{7.5)}$ for $30 \mathrm{~min}$ at $25^{\circ} \mathrm{C}$. 6-MNA $(100 \mu \mathrm{M})$ and other reagents of the incubation medium were added and the reaction was carried out as described above.

Data Analysis Kinetic data were analyzed by MichaelisMenten and Eadie-Hofstee plots. Kinetic analysis was performed by inspection of the Eadie-Hofstee plots and considering the data as monophasic kinetics. The following equation was used:

$$
V=V_{\max } \cdot S /\left(K_{\mathrm{m}}+S\right)
$$

Lineweaver-Burk plots were also used to determine values of $K_{\mathrm{m}}$ and $V_{\max }$ of diclofenac activity in the absence and presence of inhibitors as well as the inhibition types for 6-MNA. $K_{\mathrm{i}}$ value was derived from Dixon plots.

All experiments were performed in triplicate and the mean values for each data point were used for analysis. Enzyme kinetic parameters $\left(K_{\mathrm{m}}, V_{\max }\right)$ were estimated by curve fitting of metabolite formation rate data by the single enzyme Michaelis-Menten equation. All graphical analysis was performed by non-linear regression using MULTI program. ${ }^{16)}$

Analyses for correlations and statistical significances were carried out using EXCEL statistics software (Esumi Co., 


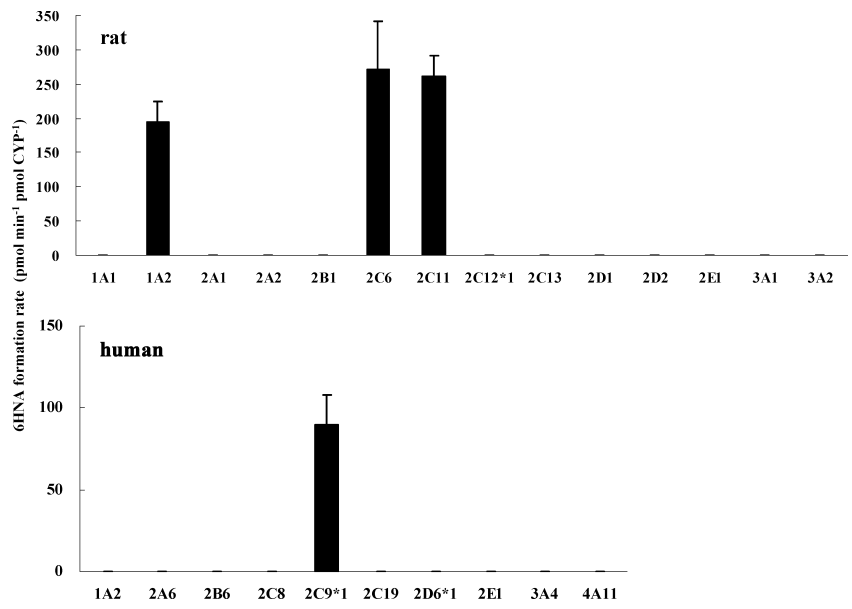

Fig. 2. Formation of 6-HNA from 6-MNA by cDNA-Expressed CYPs Each bar represents the mean \pm S.D. of 3 determinations.

Ltd., Tokyo, Japan).

\section{RESULTS}

6-HNA Formation Activity in Microsomes from Baculovirus-Infected Insect Cells Expressing Human and Rat CYP Isoforms Figure 2 shows the catalytic activities of the recombinant human CYP isoforms with respect to the formation of 6-HNA from 6-MNA with $200 \mu$ m 6-MNA. Screening of recombinant human CYPs for metabolic activity indicated that only $\mathrm{CYP} 2 \mathrm{C} 9$ contributes to the 6-O-demethylation of 6MNA (Fig. 2 human). CYP2C6 showed substantial 6-HNA formation in the recombinant rat CYP isoforms at $200 \mu \mathrm{M} \mathrm{6-}$ MNA. CYP1A2 and CYP2C11 were also found to catalyze formation of 6-HNA, comparable in activity to CYP2C6. The other recombinant CYP isoforms exhibited negligible activity (Fig. 2 rat).

Kinetic Analyses of the 6-HNA Formation from 6-MNA by Human and Rat Liver Microsomes and cDNA-Expressed CYP Isoforms The kinetics of the NADPH-dependent metabolisms of 0.05 to $4 \mathrm{~mm} 6-\mathrm{MNA}$ to its metabolite 6-HNA was examined with both pooled human and pooled rat liver microsomal preparations (Fig. 3). With both liver microsomes, analysis of Eadie-Hofstee plots revealed that 6-HNA formation exhibited monophasic enzyme kinetics. The results of the kinetic analysis of 6-HNA formation by the human and rat liver microsomes and cDNA-expressed CYP isoforms examined are shown in Table 1. The 6-Odemethylation of 6-MNA, resulting in formation of 6-HNA, was apparently monophasic in the pooled human and rat microsomes, and the data were best-fitted to a one-site binding model indicating that each reaction occurred in a manner of simple Michaelis-Menten kinetics. In addition, the slopes were not markedly different between the pooled microsomes in humans and those in rat; the apparent $K_{\mathrm{m}}$ values for the 6HNA formation in the pooled human microsomes resembled those in the pooled rat microsomes $(0.64 \mathrm{~mm}$ versus $0.72 \mathrm{~mm}$ ). For CYP2C9, the estimate of $K_{\mathrm{m}}$ was $0.31 \mathrm{~mm}$ and that of $V_{\max }$ was $4.77 \mathrm{nmol} / \mathrm{min} / \mathrm{mg}$ protein. The estimated $K_{\mathrm{m}}$ values for 6-O-demethylation of 6-MNA by $\mathrm{CYP} 2 \mathrm{C} 6$ and CYP2C11 were 0.33 and $0.14 \mathrm{~mm}$, and $V_{\max }$ values were 0.95 and $2.29 \mathrm{nmol} / \mathrm{min} / \mathrm{mg}$ protein, respectively. Reliable esti-
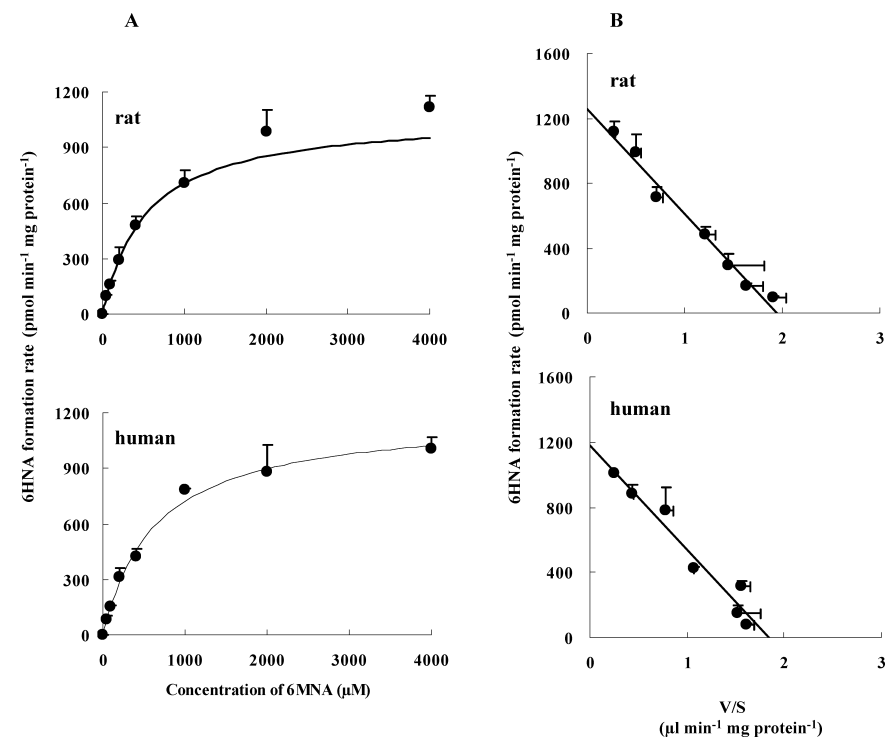

Fig. 3. Michaelis-Menten Plots (A) and Eadie-Hofstee Plots (B) for 6HNA Formation from Pooled Rat and Human Liver Microsomes

Each point represents the mean \pm S.D. of 3 determinations.

Table 1. Kinetic Analysis of the Oxidation of 6-MNA by Human and Rat Liver Microsomes and cDNA-Expressed CYPs

\begin{tabular}{lccc}
\hline \hline Enzyme source & $\begin{array}{c}K_{\mathrm{m}} \\
(\mathrm{mM})\end{array}$ & $\begin{array}{c}V_{\text {max }} \\
\left(\mathrm{nmol} \mathrm{min}^{-1}\right. \\
\left.\mathrm{mg} \mathrm{protein}^{-1}\right)\end{array}$ & $\begin{array}{c}\mathrm{Cl}_{\text {int }} \\
\left(\mu 1 \mathrm{~min}^{-1}\right. \\
\left.\mathrm{mg} \mathrm{protein}^{-1}\right)^{a)}\end{array}$ \\
\hline Human liver pooled & $0.64 \pm 0.03$ & $1.17 \pm 0.03$ & $1.8 \pm 0.0$ \\
CYP2C9 & $0.31 \pm 0.04$ & $4.77 \pm 0.78$ & $15.2 \pm 0.9$ \\
Rat liver pooled & $0.72 \pm 0.11$ & $1.31 \pm 0.07$ & $1.8 \pm 0.2$ \\
CYP2C6 & $0.33 \pm 0.03$ & $0.95 \pm 0.10$ & $2.9 \pm 0.1$ \\
CYP2C11 & $0.14 \pm 0.03$ & $2.29 \pm 0.21$ & $16.6 \pm 1.9$ \\
\hline
\end{tabular}

Data are the mean \pm standard deviation $(n=3) . \quad$ a) $\mathrm{Cl}_{\mathrm{int}}=V_{\max } / K_{\mathrm{m}}$.

mates of $K_{\mathrm{m}}$ and $V_{\max }$ could not be obtained for CYP1A2 owing to the nonsaturable nature of this reaction over the range of concentrations studied.

Correlation between 6-MNA Demethylation and Diclofenac 4'-Hydroxylation Activities by Liver Microsomes from Six Human Samples The results above suggested that CYP2C9 is the most important isoform in 6-HNA formation from 6-MNA by human liver microsomes. Correlation between diclofenac 4 '-hydroxylation and rate of formation of 6-HNA was compared in liver microsomes from six human samples. We found that there was a significant correlation $\left(r^{2}=0.951, p<0.001\right)$ between diclofenac and 6-MNA oxidation activity (Fig. 4), despite the small sample size.

Chemical Inhibition Studies The effects of co-incubation of chemical inhibitors on the 6-HNA formation from 6MNA are shown in Fig. 5. NBI and SKF525A, prototypic CYP inhibitors, inhbited the 6-HNA formation in both rat and human liver microsomes. However, thiourea and methimazole, prototypic FMO inhibitors, did not inhibit 6-HNA formation in human liver microsomes. In the rat microsomes, although methimazole inhibited the 6-HNA formation, thiourea, which is highly selective for FMOs compared to methimazole, had little inhibitory effct on the 6-HNA formation. Sulfaphenazole, a specific inhibitor of CYP2C9, was a potent inhibitor of 6-HNA formation in pooled human liver 


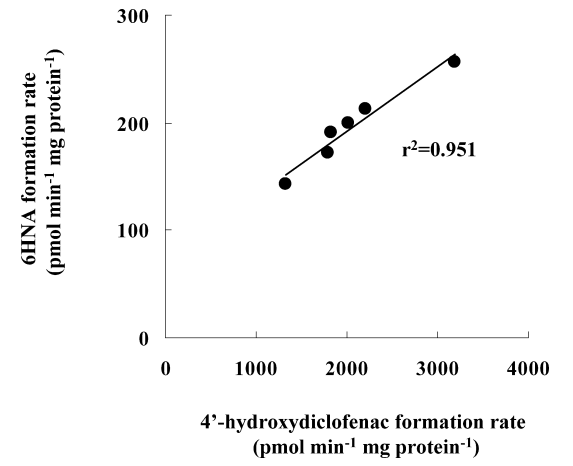

Fig. 4. Correlation between the Diclofenac 4'-Hydroxylation Activity (CYP2C9) and 6-HNA Formation in Six Human Liver Microsomal Samples

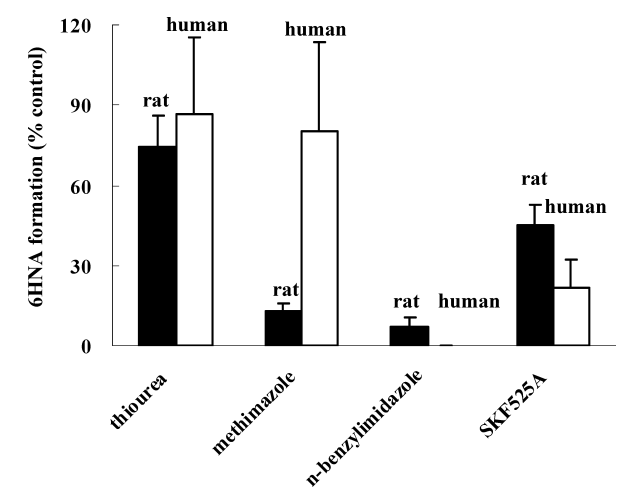

Fig. 5. Inhibition of 6-HNA Formation from 6-MNA by Co-incubation with FMO CYP-Selective Compounds in Human and Rat Liver Microsomes

Each bar represents the mean \pm S.D. of 3 determinations.

microsomes; the mean inhibition produced was about $90 \%$ of the control (Fig. 6A). However, sulfaphenazole did not inhibit 6-HNA formation in rat liver microsomes. Furafylline, $S$-mephenytoin, quinidine, diethyldithiocarbamate, troleandomycin and ketoconazole had little inhibitory effects on 6MNA metabolism in both rat and human liver microsomes (data not shown). Figure 7 shows the effect of cimetidine, inhibitors of CYP2C11/2C6, on 6-MNA metabolism in male and female rat liver microsomes. Cimetidine at $50 \mu \mathrm{M}$ decreased the 6-MNA formation by about $30 \%$ and $60 \%$ of control in male and female rat liver microsomes, respectively. The rate of formation of 6-HNA of control in male rat liver microsomes was about 1.5 -fold greater than in the female rat liver microsomes.

Representative Lineweaver-Burk plots of the diclofenac 4'-hydroxylation activity inhibition data are shown in Fig. $8 \mathrm{~A}$. Four concentrations of 6 -MNA $(0,0.25,0.5,1.0 \mathrm{~mm})$ decreased the $V_{\max }$ of the diclofenac 4'-hydroxylation, whereas the $K_{\mathrm{m}}$ was increased from a control value of 4.35 to 5.43 , 5.44 and $8.47 \mu \mathrm{M}$, respectively. This indicates that 6-MNA is a mixed-type inhibitor of diclofenac 4 '-hydroxylation activity. The $K_{\mathrm{i}}$ value for 6-MNA derived from Dixon plots was $724.2 \mu \mathrm{M}$ (Fig. 8B).

Chemical Activation Studies Dapsone, a specific activator of CYP2C9, was an effective activator of 6-HNA formation in the pooled human microsomes (Fig. 6B). Mean activity elicited by dapsone was about 4-fold higher than the control value. However, the production of 6-HNA was not promoted by dapsone in the rat microsomes.

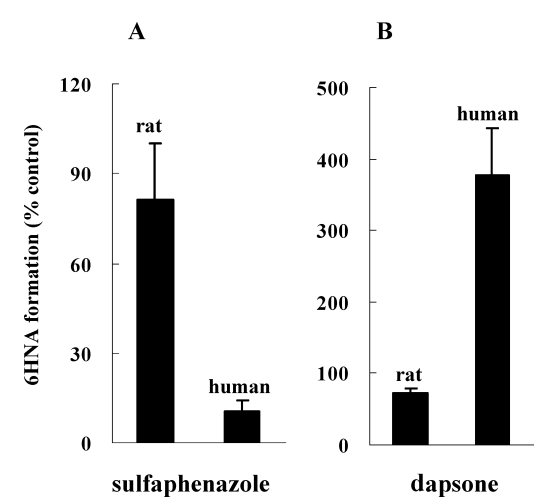

Fig. 6. The Effects of Sulfaphenazole (A) and Dapsone (B) on the 6-HNA Formation from 6-MNA in Human and Rat Liver Microsomes

Each bar represents the mean \pm S.D. of 3 determinations.

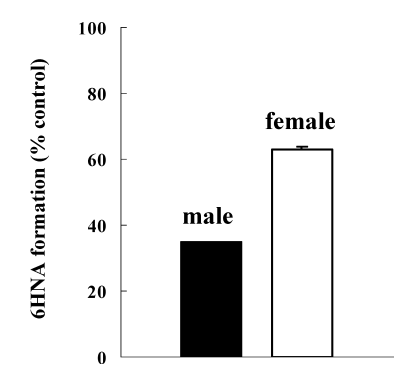

Fig. 7. The Effects of Cimetidine on the 6-HNA Formation from 6-MNA in Male and Female Rat Liver Microsomes

Each bar represents the mean \pm S.D. $(n=3)$.

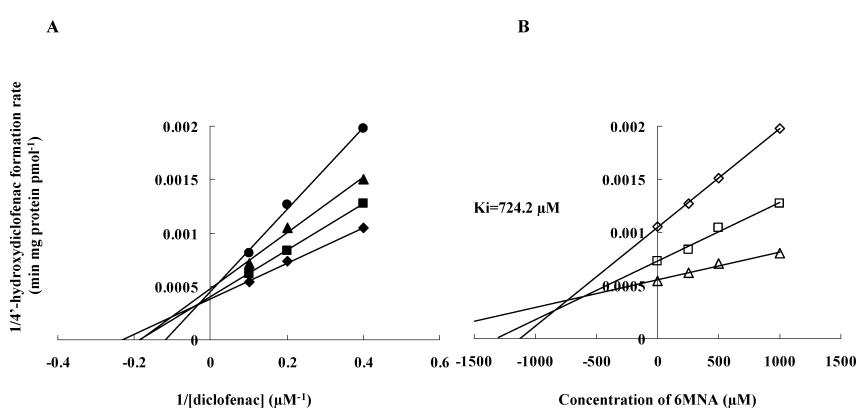

Fig. 8. Lineweaver-Burk Plots at $0 \mu \mathrm{M}(\bullet), 250 \mu \mathrm{M}(\boldsymbol{\bullet}), 500 \mu \mathrm{M}(\mathbf{\Delta})$ and $1000 \mu \mathrm{M}(\bullet)$ Concentrations of 6-MNA (A) and Dixon Plots at $2.5 \mu \mathrm{M}(\diamond)$, $5 \mu \mathrm{M}(\square)$ and $10 \mu \mathrm{M}(\triangle)$ Concentrations of Diclofenac (B)

Each point represents the mean $(n=3-4)$.

Analysis of Lineweaver-Burk plots confirmed the effects of dapsone on $K_{\mathrm{m}}$ and $V_{\max }$ of 6-HNA formation from 6MNA (data not shown). Increasing concentration of dapsone caused a decrease in the slope of each linear regression line, which was suggestive of a decrease in $K_{\mathrm{m}}$ and an increase in $V_{\max }$ for the 6-HNA formation.

Immunoinhibition Studies Figure 9A shows the effects of anti-NADPH-P450 reductase and anti-CYP antibodies on the formation of 6-HNA in liver microsomes from humans and rats. In the rat and human microsomes, the addition of antibodies against rat NADPH-P450 reductase reduced the 6HNA formation by about $20 \%$ compared with that of the control (Fig. 9A). In addition, the anti-CYP2C11 antibody had a potent inhibitory effect on the 6-HNA formation $(<25 \%)$, whereas anti-CYP1A2 antibody had little effect on this activity (Fig. 9B rat). In contrast, the antibody raised 


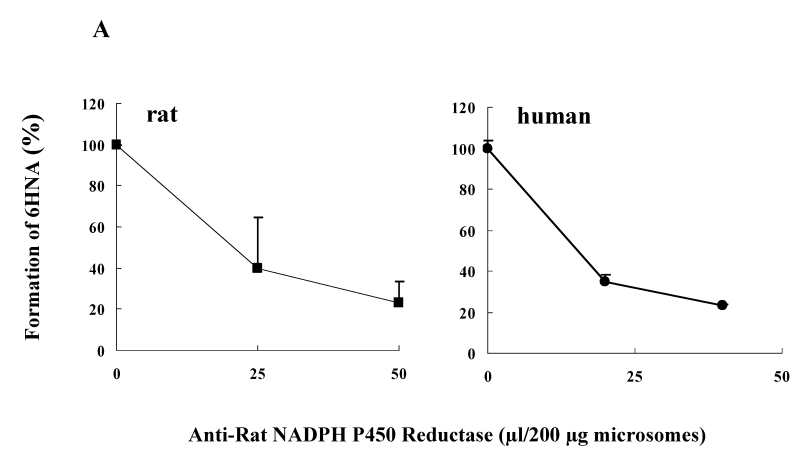

B

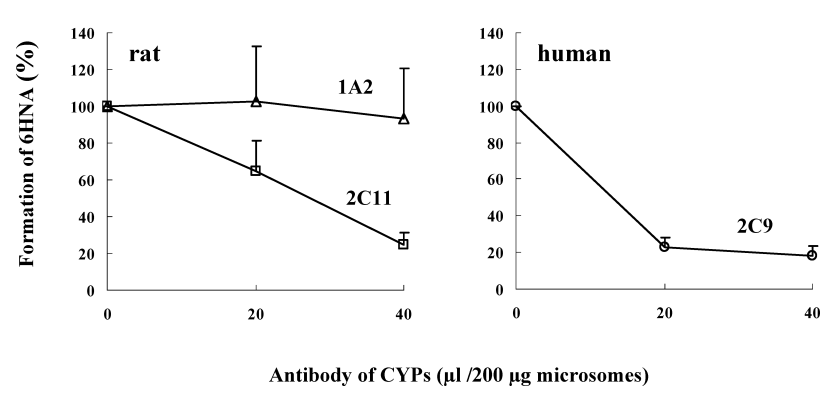

Fig. 9. The Effects of Anti-rat NADPH P450 Reductase Antibody (A) and Antibody of CYPs (B) on the 6-HNA Formation from 6-MNA in Human and Rat Liver Microsomes

Each point represents the mean \pm S.D. $(n=3)$

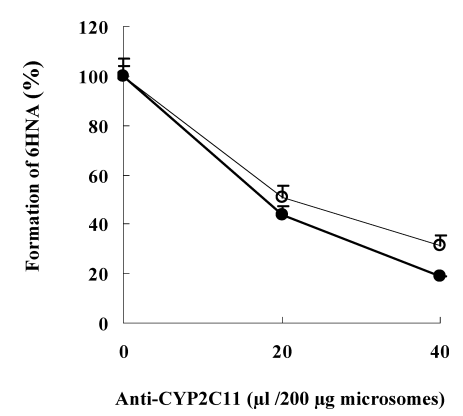

Fig. 10. The Effects of Anti-CYP2C11 Antibody on the 6-HNA Formation from 6-MNA in Male $(\bullet)$ and Female $(O)$ Rat Liver Microsomes

Each point represents the mean \pm S.D. $(n=3)$.

against human CYP2C9 inhibited the formation of 6-HNA in the human pooled liver microsomes by $80 \%$ or more (Fig. $9 \mathrm{~B}$ human).

In the male and female rat microsomes, the addition of anti-CYP2C11 antibody reduced the 6-HNA formation by about $20 \%$ compared with that of the control (Fig. 10 AntiRat CYP2C11). Anti-CYP2C11 antibody shows its cross-reactivity with CYP2C6. ${ }^{17)}$ Anti-CYP2C11 antibody was verified for its inhibitory potency and specificity using cDNAexpressed CYP isoforms. Anti-CYP2C11 had a potent inhibitory effect on the 6-HNA formation $(<25 \%)$ of the CYP2C6 as well as the CYP2C11(data not shown).

\section{DISCUSSION}

Nabumetone, which is a nonacidic NSAID, undergoes extensive metabolism to the active metabolite 6-MNA after its absorption. 6-MNA is a major circulating material, which is a potent inhibitor of prostaglandin synthesis, and is considered to be an active anti-inflammatory metabolite. ${ }^{1)}$ 6-MNA is eliminated by metabolism, principally $O$-demethylation, to yield 6-HNA in experimental animals and humans. ${ }^{3)}$ This compound has no anti-inflammatory properties and is a weak inhibitor of cyclo-oxygenase. ${ }^{1)}$ In the present study, to the best of our knowledge, we report for the first time the in vitro metabolism of 6-MNA, which is a pharmacologically active metabolite of nabumetone, in the presence of human liver microsomes as well as recombinant human CYP isoforms.

To obtain these results, we used a combination of five in vitro approaches, which can be used to determine the isoforms involved in the metabolism of 6-MNA in liver microsomes: 1) screening the metabolism of the compound with a panel of different heterologously expressed CYP; 2) kinetic analyses of $O$-demethylation activity; 3 ) correlation analysis using a panel of human liver microsomal preparations; 4) selective inhibition and stimulation of the main CYP enzymatic activities in liver microsomes with chemical compounds; and 5) immunoinhibition with antibodies.

Screening of 6-MNA metabolism with a panel of cDNAexpressed human CYP isoforms indicated that CYP2C9 has the highest enzymatic activity for the generation of 6-HNA (Fig. 2). The involvement of other CYP forms in the formation of 6-HNA is unlikely. In contrast, experiments showed that CYP2C6, CYP2C11 and CYP1A2 could metabolize 6MNA. It has been reported that these isoforms are expressed in rat liver, although CYP2C11 is a male-specific isoform. ${ }^{18-20)}$ Accordingly, it was expected that the contribution of CYP2C isoforms to 6-MNA 6-O-demethylation in liver would be considerable.

In kinetic analyses, 6-MNA metabolism conformed to single-enzyme Michaelis-Menten kinetics. Interestingly, our estimates for $K_{\mathrm{m}}$ and $V_{\max }$ values for the 6-HNA formation of the human liver microsomes were similar to those of the rat liver microsomes. That is to say, we have not provided evidence for marked species differences in the $O$-demethylation rate, at least in vitro. However, our results suggest that the $K_{\mathrm{m}}$ values for the 6-HNA formation in recombinant systems are lower than those in liver microsome preparations. It is unclear why the apparent affinity of the enzyme differs substantially between liver microsome preparations and recombinant systems, although differences in reductase levels, b5 levels and lipid composition between recombinant systems and liver microsomes may be factors that contribute to this difference. $^{21)}$ Eadie-Hofstee plots of 6-HNA formation activities in the rat pooled microsomes seemed to be monophasic. The reason for this result might be due to the similar $K_{\mathrm{m}}$ values of CYPs, perhaps CYP2C6 and CYP2C11 for the 6-HNA formation activities. ${ }^{22-24)}$

We also investigated which CYP isoform(s) is involved in the 6-O-demethylation of 6-MNA in pooled human liver microsomes. CYP2C9 was identified as the major isoform involved in the oxidation of 6-MNA by human liver microsomes on the basis of the following lines of evidence. First, the metabolism of 6-MNA into 6-HNA was found to be monophasic on Eadie-Hofstee plots, suggesting that its metabolism was mainly dependent on a single enzyme. Second, the specific CYP2C9 inhibitor sulfaphenazole significantly diminished 6-HNA formation (Fig. 6A), but on the other hand, the specific CYP2C9 stimulator dapsone ${ }^{15)}$ signifi- 
cantly activated 6-HNA formation (Fig. 6B). Third, the 6HNA formation was inhibited significantly by the addition of anti-CYP2C9 antibody (Fig. 9B). Fourth, the involvement of CYP2C9 was further demonstrated by the inhibition of CYP2C9-mediated diclofenac-4' -hydroxylation $^{25)}$ by 6-MNA (Fig. 8). Fifth, a significant correlation was observed between the 4'-hydroxy-diclofenac formation rate, a marker for CYP2C9, and 6-HNA formation from $200 \mu \mathrm{M}$ 6-MNA $\left(r^{2}=\right.$ $0.951, p<0.001)$. These data clearly indicated that CYP2C9 is mainly responsible for the 6-O-demethylation of 6-MNA in human liver microsomes.

The inhibitory potentials of 6-MNA on the clinically significant human CYPs, namely, 1A2, 2A6, 2C9, 2C19, 2D6 and $3 \mathrm{~A} 4$, were also investigated in human liver microsomes (data not shown). 6-MNA itself was a very weak inhibitor of CYP2C9 in pooled human liver microsomes when incubated for $20 \mathrm{~min}\left(K_{\mathrm{i}}=724.2 \mu \mathrm{M}\right)$, with no significant effect on the isoforms tested. It is unlikely that 6-MNA itself will significantly alter the clearance of other compounds metabolized by CYP2C9 because a repeated oral dose of nabumetone $(800 \mathrm{mg})$ results in a steady state serum-unbound concentration of about $2 \mu \mathrm{M}^{26)}$ However, a clinical study is necessary to establish the extent of interaction between 6-MNA and drugs that are CYP2C9 substrates.

In the current study, the anti-NADPH-P450 reductase $^{27)}$ inhibited the formation of 6-HNA, demonstrating that the oxidation of 6-MNA is mediated by CYPs. Between human and rat $\mathrm{CYP} 2 \mathrm{C}$ isoforms reveals differences in their expression. ${ }^{25)}$ In recombinant rat CYP systems, both CYP2C6 and CYP2C11 catalyzed the oxidation of 6-MNA. Cimetidine (CYP2C11/6 inhibitor) inhibited 6-HNA formation in both male and female rat liver microsomes. In the rat, there is a sex difference in the content of CYP2C11: mature female rats do not have $\mathrm{CYP} 2 \mathrm{C} 11$. However, both male and female rats have CYP2C6, which has high activity toward 6-MNA that CYP2C11 can metabolize. In the male and female rat microsomes, the addition of anti-CYP2C11 reduced the 6HNA formation. Anti-CYP2C11 antibody, which crossreacted with CYP2C6, inhibited the formation of 6-HNA. These results strongly suggest that CYP2C enzymes, especially CYP2C6 and CYP2C11, are the major isoforms responsible for the oxidation of 6-MNA in rat liver. 6-MNA oxidation by CYP1A2 was catalyzed to some extent, suggesting that CYP1A2 isoform is also involved in the oxidation of 6-MNA in rats. Levels of CYP isoforms in male rats are very different, with CYP2C11 accounting for 54\% of total CYP content but CYP1A2 being expressed at much lower levels $(2 \%)$ in untreated rat liver samples. ${ }^{18-20)}$ In addition, antiCYP1A2 antibody did not clearly inhibit the formation of 6HNA. Therefore, CYP1A2 is unlikely to be the principal enzyme mediating 6-MNA oxidation in rats.

In summary, in vitro metabolism studies showed that CYP2C9 has the highest catalytic activity of 6-MNA metabolism in human liver microsomes. In contrast, metabolism of
6-MNA is mediated mainly by CYP2C6 and CYP2C11 in rat liver microsomes. The comparative data presented here revealed the different CYP isoforms in two species, and provided the useful information of selecting rats as an animal model for in vitro and in vivo experiments on nabumetone.

Acknowledgement The authors thank Ms. Yuko Sato for expert technical assistance.

\section{REFERENCES}

1) Davies N. M., Clin. Pharmacokinet., 33, 403-416 (1997).

2) Mangan F. R., Flack J. D., Jackson D., Am. J. Med., 83 (Suppl. 4B), 6-10 (1987).

3) Haddock R. E., Jeffery D. J., Lloyd J. A., Thawley A. R., Xenobiotica, 14, 327-337 (1984).

4) Kobylinska K., Balinska M., Kobylinska M., J. Pharm. Biomed. Anal., 32, 323-328 (2003).

5) Mikami E., Goto T., Ohno T., Matsumoto H., Nishida M., J. Pharm. Biomed. Anal., 23, 917-925 (2000).

6) Newton D. J., Wang R. W., Lu A. Y. H., Drug Metab. Dispos., 23, $154-158$ (1995).

7) Rodrigues A. D., Biochem. Pharmacol., 57, 465-480 (1999).

8) Rodrigues A. D., Robert E. F., Mulford D. J., Yao Y., Ouellet D., Drug Metab. Dispos., 25 , 623-630 (1997).

9) Suzuki A., Iida I., Tanaka F., Akimoto M., Fukushima K., Tani M., Ishizaki T., Chiba K., Drug Metab. Dispos., 27, 1254-1259 (1999).

10) Bourie M., Meunier V., Berger Y., Fabre G., J. Pharmacol. Exp. Ther, 277, $321-332$ (1996).

11) Levine M., Law E. Y., Bandiera S. M., Chang T. K., Bellward G. D., J. Pharmacol. Exp. Ther, 284, 493-499 (1998).

12) Szakács T., Veres Z., Vereczkey L., Pol. J. Pharmacol., 53, 11-19 (2001).

13) Grothusen A., Hardt J., Bräutigam L., Lang D., Böcker R., Arch. Toxicol., 71, 64-71 (1996).

14) Mani C., Hodgson E., Kupfer D., Drug Metab. Dispos., 21, 657-661 (1993).

15) Hummel M. A., Dickmann L. J., Rettie A . E., Haining R. L., Weaver R. J., Tracy T. S., Biochem. Pharmacol., 67, 1831-1841 (2004).

16) Yamaoka K., Tanigawara Y., Nakagawa T., Uno T., J. PharmacobioDyn., 4, 879-885 (1981).

17) Imaoka S., Terano Y., Funae Y., Arch. Biochem. Biophys., 278, 168 178 (1990).

18) Imaoka S., Yamada T., Hiroi T., Hayashi K., Sakaki T., Yabusuki Y., Funae Y., Biochem. Pharmacol., 51, 1041-1050 (1996).

19) Nedelcheva D., Gut I., Xenobiotica, 24, 1151-1175 (1994).

20) Guengerich F. P., Dannan G. A., Wright S. T., Martin M. V., Kaminski L. S., Biochemistry, 21, 6019-6031 (1982).

21) Tracy T. S., Marra C., Wrighton S. A., Gonzalez F. J., Korzekwa K. R., Eur. J. Clin. Pharmacol., 52, 293-298 (1997).

22) Nakajima M., Kobayashi K., Shimada N., Tokudome S., Ymamoto T., Kuroiwa K., Br. J. Clin. Pharmacol., 46, 55-62 (1998).

23) Yoshii K., Kobayashi K., Tsumuji M., Tani M., Shimada N., Chiba K., Life Sci., 67, 175-184 (2000).

24) Akimoto M., Iida I., Itoga H., Miyata A., Kawahara S., Kohno Y., Eur. J. Drug Metab. Pharmacokinet., 29, 176-186 (2004).

25) Bogaads J. J. P., Bertrand M., Jackson P., Oudshoorn M. J., Weaver R. J., Bladeren P. J., Walther B., Xenobiotica, 30, 1131-1152 (2000).

26) Takasugi K., Tokudome S., Shimada N., Yamazaki H., Yokoi T., Inflammation, 27, 359-361 (1988).

27) Nakamura A., Hirota T., Morino A., Shimada T., Uematsu T., Xenobiotica, 52, 995-1003 (1997). 